\title{
O-DSR: OPTIMIZED DSR ROUTING PROTOCOL FOR MOBILE AD HOC NETWORK
}

\author{
Rjab Hajlaoui $^{1}$, Sami Touil ${ }^{2}$ and Wissem achour ${ }^{3}$ \\ ${ }^{1}$ Department of Computer Sciences, Hail University, Hail, KSA \\ ${ }^{2}$ Sami Touil, Qassim University, Qassim, KSA \\ ${ }^{3}$ Department of Computer Sciences, Qassim University, KSA
}

\begin{abstract}
A MANET is an autonomous collection of mobile users that communicate over relatively bandwidth constrained wireless links. When designing mobile ad hoc networks, several interesting and difficult problems arise because of the shared nature of the wireless medium, limited transmission power (range) of wireless devices, node mobility, and battery limitations.

This paper aims at providing a new schema to improve Dynamic Source Routing (DSR) Protocol. The aim behind the proposed enhancement is to find the best route in acceptable time limit without having broadcast storm. Moreover, O-DSR enables network not only to overcome congestion but also maximize the lifetime of mobile nodes. Some simulations results show that the Route Request (RREQ) and the Control Packet Overhead decrease by $15 \%$ when O-DSR is used, consequently. Also the global energy consumption in $O-D S R$ is lower until to $60 \%$, which leads to a long lifetime of the network.
\end{abstract}

\section{KEYWORDS}

Optimized DSR; Routing protocol; broadcasting; Overhead; MANET.

\section{INTRODUCTION}

Mobile Ad hoc Network (MANET), is a set of autonomous mobile units forming a temporary network without any fixed infrastructure $[13,20]$.

To stay connected, each entity plays the role of a router and partly share the responsibility of routing data $[16,18]$.

This handling decrease the life time of networks and causes a RREQ and Control Packet Overhead. Following this, the goal of our work is to improve the behaviour of DSR by using a robust algorithm which optimizes the operation of data dissemination.

To put it differently, the zone containing destination must be focused before starting the mechanism of route discovery. This proactive step reduces the broadcasting overhead. 
In the suggested diagram the aim is to overcome broadcast storm by establishing in each branch of the network one list containing the nodes that form this branch in order to identify the search area. In this way and with a simple test, we will eliminate the branches which do not contain the destination and the broadcasting of RREQ will be restricted only to remaining areas.

The main advantage of this approach is to reduce the network congestion by minimizing the number of RREQ packets.

The rest of this paper is divided into five sections. Section 2 gives a brief description of the adhoc routing protocol, specially DSR, section 3 presents the related work, Section 4 describes our DSR optimization. Section 5 elaborates on the simulation setup and the experiment result. Section 6 concludes the paper.

\section{AD-HOC ROUTING PROTOCOLS}

Several routing protocols have been developed for ad hoc mobile networks [10, 7]. In these networks there is no fixed topology due to the mobility of nodes, interference, multipath propagation and path loss. Hence a dynamic routing protocol is needed for these networks to function properly. Many Routing protocols have been developed for accomplishing this task [14].

A classification of the most important routing protocol is presented in figure 1 .

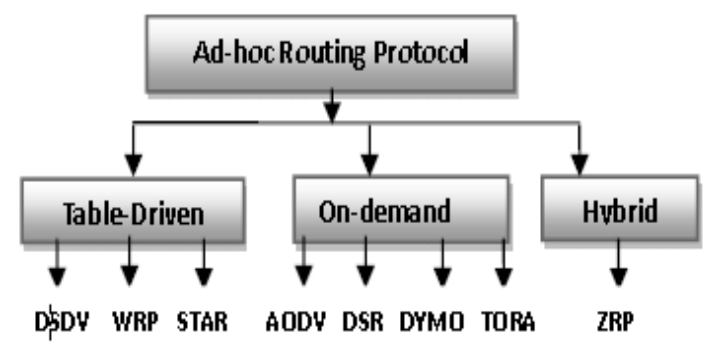

Figure 1. Categorization of ad-hoc routing protocol

The DSR protocol has increased traffic overhead, so why it is applicable to a relatively small amount of nodes. Otherwise, managing big network deal with quick congestion and reduce the life time of network [8]. Based on those shortages, we will try to enhance the quality of results given by this protocol.

\section{DSR ROUTING PROTOCOL}

The Dynamic Source Routing (DSR) is a reactive unicast routing protocol that makes use of source routing algorithm, where each data packet contains complete routing information to reach its dissemination $[4,9]$.

The DSR protocol is composed of two mechanisms that work together to allow the discovery and maintenance of source routes in the ad hoc network (eg. figure 2):

- Route Discovery is the mechanism by which a node $S$ wishing to send a packet to a destination node D obtains a source route to D. Route Discovery is used only when $S$ attempts to send a packet to $\mathrm{D}$ and does not already know the route to $\mathrm{D}$. 
- Route Maintenance is the mechanism by which node $\mathrm{S}$ is able to detect while using a source route to $\mathrm{D}$. If the network topology has changed, this means that it can no longer use its route to $\mathrm{D}$ because the link along the route no longer works. When Route Maintenance indicates that the source route is broken, $\mathrm{S}$ can use any other route that knows the route to $\mathrm{D}$, or invoke Route Discovery again to find a new one. Route maintenance is used only when $\mathrm{S}$ is actually sending packets to $\mathrm{D}[3]$.
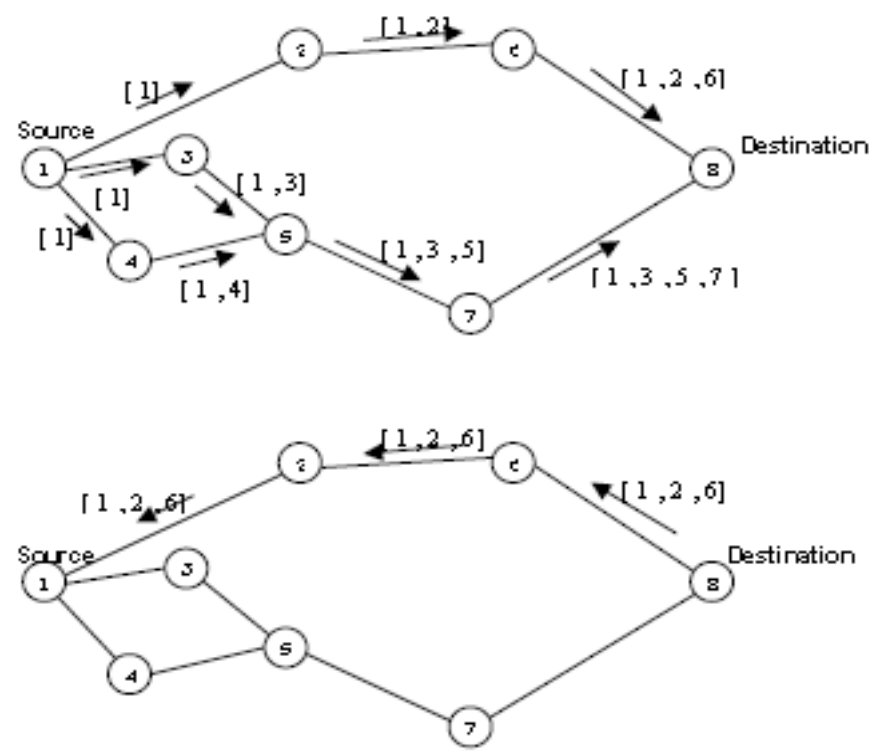

Figure 2. Discovery and maintenance of routes in DSR

Several optimizations to this basic protocol have been proposed and have been evaluated to be very effective by the authors of the protocol. Some of them are:

a) Data Salvaging - If an intermediate node encounters a broken link and has an alternate route to the destination in its cache, it can try to salvage the packet by sending it via the route from its cache.

b) Gratuitous Replies - When a node overhears a packet not addressed to itself, it checks to see if the packet could be routed via itself to gain a shorter route. If so, the node sends a gratuitous reply to the source of the route with this new better route.

c) Route Snooping - A node that overhears a data packet and does not have the route indicated in the packet's header in its own cache, adds the route to its cache for future use [24].

\section{RELATED WORK}

In [11] three power-oriented routing protocols based on the DSR protocol were developed, the protocols were respectively: MDR, LEAR and EDDSR.

Minimum Drain Report (MDR): This protocol offers minimum drain rate as a new metric to use in conjunction with the remaining battery capacity to predict life time of nodes according to the current traffic conditions [5], but it does not guarantee that the total transmission energy is minimized over a chosen route. 
Local Energy-Aware Routing (LEAR): This enhancement aims to balance energy consumption with shortest routing delays, takes into account a node"s willingness to participate in the routing path which is based on its remaining battery power. Furthermore the destination does not wait to reply which is the source of efficient use of route cache [21]

Energy Dependent mechanism (DSR EDDSR): It tries to avoid the use of a low power node battery, using information related to the residual energy in the route discovery, whereas each node determines its willingness to participate in routing packets based on its energy level. However, considering the latter as single factor may not be sufficient in all cases, because it provides no link availability among nodes of a higher energy.

Selected routes in this case can then be exposed to more failures of links that consume more energy to turn during the process of maintenance of roads [12, 27].

In [15] a new GSM-DSR (Generalized Salvaging Mechanism for DSR) was proposed, these optimizations are subdivided in two components and consist in generalizing the data packets salvaging technique of DSR. On the other hand, the second contribution aims at reinforcing the salvaging optimization, by providing it with a reliable support. In [26], A Novel Optimization of Route Discovery in Dynamic Source Routing (DSR) Protocol for MANET was proposed. The optimization aims to minimize the number of cached route replies (RREP), which is a significant source of overhead for the dynamic source routing (DSR) protocol [1].

\section{O-DSR: OPTIMIZED DSR ROUTING PROTOCOL}

\subsection{Contribution}

In this section we explain the enhancement attached to DSR protocol in order to get the best route and to deliver data from source to destination in the intended deadline without having broadcasting storm. In contrast to the process of route selection in the DSR protocol which selects the shortest route to the destination using automatically route discovery and route maintenance, for example if in an average each node has $\mathrm{x}$ neighbours and there are $\mathrm{y}$ hops from source to destination and $\mathrm{R}$ is the total number of RREQ packets then basic DSR

requires, $\mathrm{R}=\mathrm{x}^{*} \mathrm{y}$ RREQ packets to be broadcasted [22].

To overcome this broadcasting storm our approach defines the forwarding zone containing destination as the first step in order to reduce the RREQ and packet control overhead. Our extension contains two phases.

\section{Phase 1: Lists Construction:}

\section{Algorithm of List construction:}

- $\quad S==$ size of the network. (number of nodes)

- N: node

- Li: list $\mathrm{i}$

- $\quad \mathrm{Nbn}(\mathrm{Ni})$ : Number of neighbours of node $\mathrm{Ni}$

- $\quad \mathrm{I}==0$

For $\mathrm{j}=1$ to $\mathrm{s}$

\{

$\mathrm{Li}=\{\}$ 


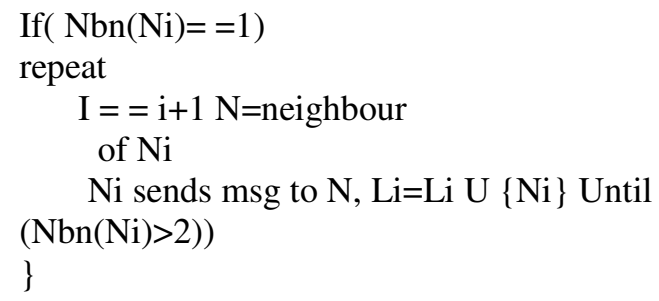

\section{Phase2: Update of lists:}

Causes of network mobility, an update procedure must be applicated when any node from any list $\mathrm{Li}$ establishes or releases a connection. This update phase can be explained as follows: If neighbour number of the node $\mathrm{Ni}$ from list Li changes, then this node sends an alert message to their neighbours. The node receiving this alert must delete any list containing $\mathrm{Ni}$ and passes the same order to its neighbours (eg, Figure 3)

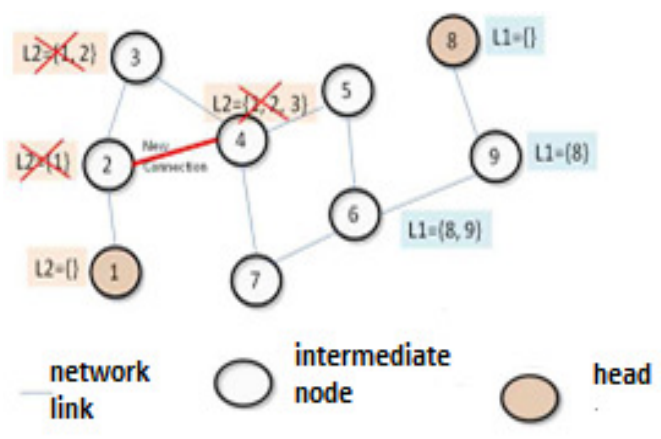

Figure 3. List Update

\subsection{Validation}

- For each node $\mathrm{Ni}$ of the network, if it has only one neighbour $(1,8)$, this node will be the head of a new list $\mathrm{Li}$ and $\mathrm{Li}=\mathrm{Li} \mathrm{U} \mathrm{Ni}(\mathrm{L} 1=\{8\}, \mathrm{L} 2=\{1\})$ (e.g., Figure 4)

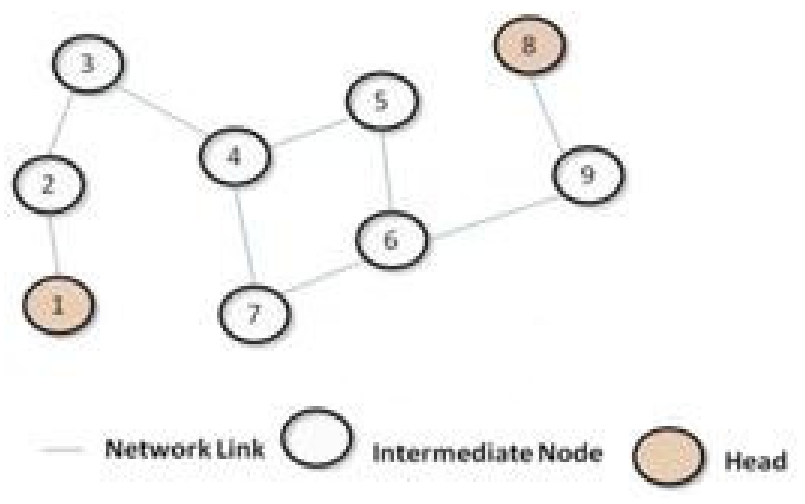

Figure 4. Lists Construction 
This list will be extended from one node to another when the number of neighbours of the new node is $<3$,

L1 will be $\{8,9\}$ and L2 will be $\{1,2,3\}$ (eg, Figure 5)

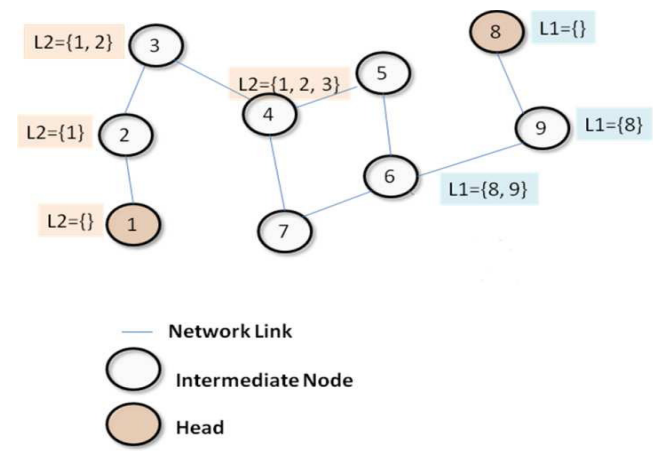

Figure 5. List Extension

\section{Example}

When node 4 wants to communicate with node1, the broadcasting starts with a small test as follows:

If node $1 \in \mathrm{L} 2$, then data will be oriented directly using $\mathrm{L} 2$ as the best and unique route.

Else \{

- $\quad$ All nodes within L2 will be eliminated from broadcasting.

- $\quad$ RREQ will be sent to node 5 and node7

- $\quad$ In node 6: If node $1 \in \mathrm{L} 1$, then $\mathrm{L} 1$ will be the route to transmit data. \}

Based on DSR protocol, we propose a new technique that considers the forked part of the network as a cluster. So the broadcasting will be focused on one part instead of treating the entire network.

Using this technique RREQ will be confined in a small part of network and the broadcasting can be significantly reduced.

Our solution is also able to find the best path between sources and destinations in a short time and to reduce the activities of many nodes to save energy and storage memory. When broadcasting decreases, we will obtain low collision and the performance of network will rise. (eg, Figure 6)

a- Using DSR

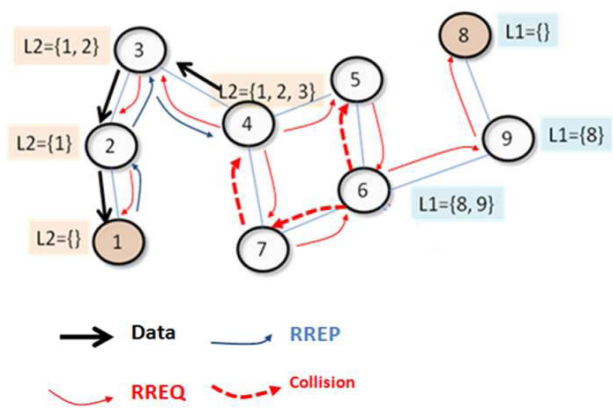




\section{Using $O-D S R$}

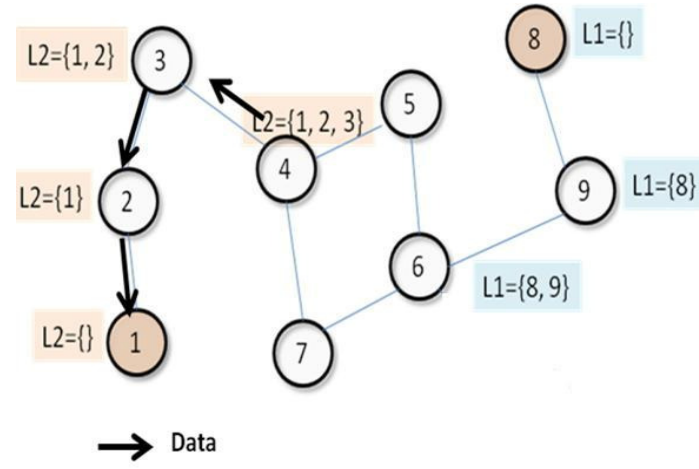

Figure 6. Comparison of data broadcasting from node 4 to node 1

\subsection{Simulation}

All the simulations were performed with network simulator NS2 [25]. The results obtained after our enhancement are compared with the reactive routing protocol DSR. The performances of ODSR are elevated in the measurement of the four well known metrics: RREQ Overhead, Control Packet Overhead, Packet Delivery Fraction and Consumed Energy per Packet [19, 17, 6]

\subsubsection{RREQ Overhead (RRO)}

The total number of routing request packets transmitted during the simulation, each transmission of the packet (each hop) counts as one transmission.

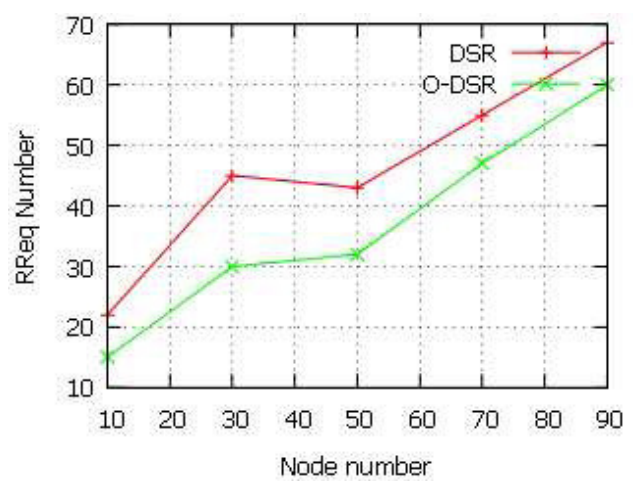

Figure 7. RREQ Overhead: O-DSR vs DSR protocol

It is clear in figure 7 that the RREQ overhead value in our solution is lower than DSR routing protocol in all experiments. This reduction is the consequence of improvement given in the mechanism of Route Discovery, since in many times the route from Source(S) to Destination (D) may exist in advance and in this case no RREQ is sent.

For example with a density of 30 nodes, the improvement is $(45-35) / 45=10 \%$. This improvement decreases with a network of 90 nodes and will be equal to $((74-67) / 74=9.5 \%$. 


\subsubsection{Control Packet Overhead (CPO)}

The total number of control packets transmitted during the simulation.

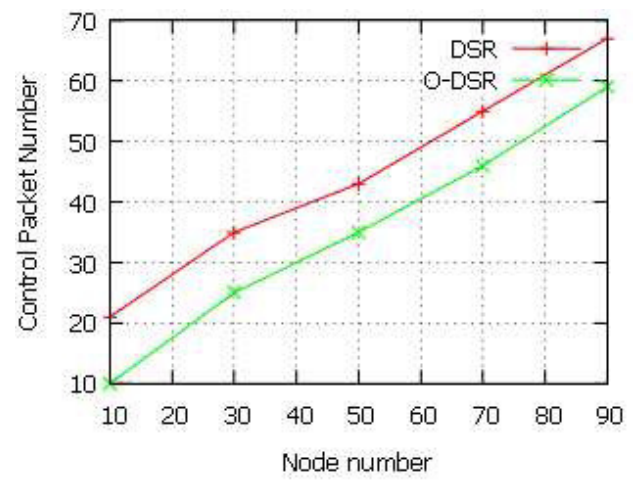

Figure 8. Control Packet Overhead: O-DSR vs DSR protocol

The proposed solution can find the destination effectively and speedily compared with DSR protocol, therefore, it is imperative that the packet control overhead decreases because of the use of less RREQ number .

For instance, for a network of 30 nodes as density, the improvement in control packet overhead is $(35-30) / 100=5 \%$.

\subsubsection{Packet Delivery Fraction (PDF)}

The ratio of data packets well received by destination nodes to that generated by source nodes.

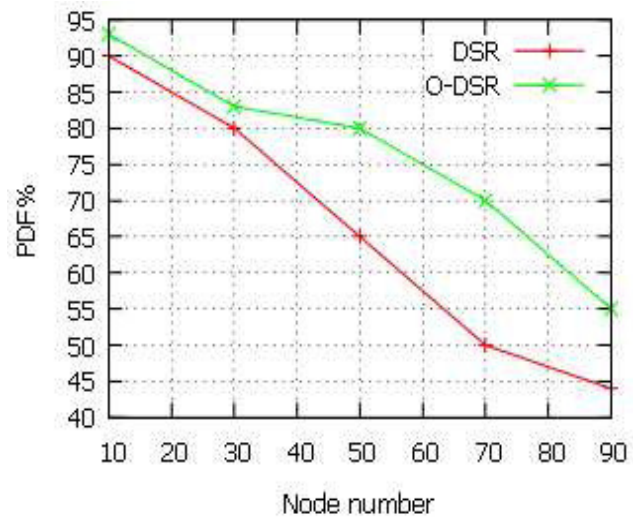

Figure 9. Packet Delivery Fraction

With O-DSR and during the research of the best way from source to destination we don "t treat the totality of the network but we seek in a present zone in advance, therefore the number of message circulating in the network is much lower than DSR which is characterized by a high congestion queues communication interfaces. For this reason the PDF of O-DSR was always better we also note that with the increase of the density of the network, the PDF of both protocols decreases. 


\subsubsection{Consumed Energy per Packet (CEP)}

The ratio of global consumed energy to the number of data packets received.

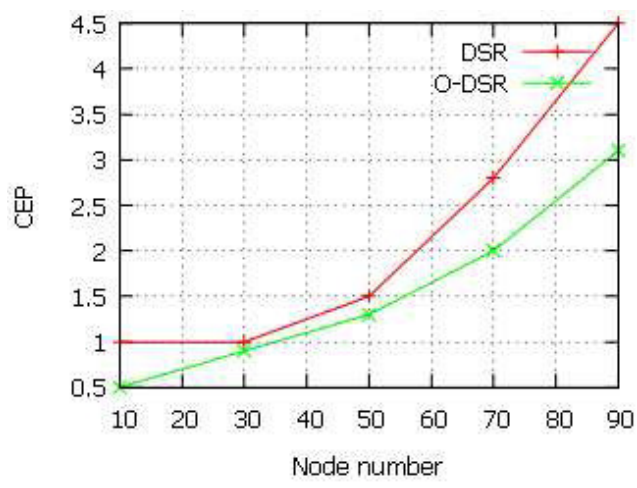

Figure 10. Consumed Energy per Packet

With the increase of the density of the network we note a remarkable rise of the number of the exchanged packets. Thus the risk of the interferences increases what justifies the increase of the CEP for both protocols.

For all the conditions of density, the CEP of O-DSR was inferior to that of the DSR because ODSR always generates less of overhead than DSR and the nodes activities is less. By consequence, the global energy consumption in O-DSR remains lower and the network lifetime will increase. Finally, we can conclude that O-DSR clearly improves DSR by reducing the number of exchanged request for discovering route and for control. This improvement has a direct effect on reducing the consumed energy for routing.

\section{CONCLUSIONS}

DSR is a widely used routing protocol for mobile ad hoc networks. But, it has a very low delivery rates and poor performance in lightly loaded networks with high node mobility [2, 23]

The innovation in our solution is the division of the network into different parts in a very simplified way, we divides the totality of the graph into different zones based on the branches of this graph.

Before moving from one branch to another, a simple test will be done to turn effectively the broadcasting of RREQ. So the network congestion status, the energy level and the survival time of network become much better. 
International Journal of Wireless \& Mobile Networks (IJWMN) Vol. 7, No. 4, August 2015

\section{REFERENCES}

[1] A. Singh et al. (2010) "A Survey of Energy Efficient Routing Protocols for Mobile Ad-hoc Networks". (IJCSE) International Journal on Computer Science and Engineering Vol. 02, No. 09, 3111-3119.

[2] B. Rajendra , A. Mathur. (2005) "Analysis of the Dynamic Source Routing Protocol for Ad Hoc Networks", Workshop on Next Generation Wireless Networks.

[3] D- B. Johnson et al. (2000) “DSR: The Dynamic Source Routing Protocol for Multi-Hop Wireless Ad Hoc Networks", Computer Science Department, Carnegie Mellon University, Pittsburgh.

[4] D. Johnson et al. (2002) "The dynamic source routing protocol for mobile ad hoc networks", InternetDraft, draft-ietf-manetdsr-07.

[5] D. Kim et al. (2002) " Power-Aware Routing Based on The Energy Drain Rate for Mobile Ad Hoc Networks", In Proc. of IEEE International Conference on Computer Communications and Networks, pages 565-569.

[6] Dilli Ravilla et al . (2011) "HYBRID ROUTING PROTOCOLS FOR AD HOC WIRELESS NETWORKS", International Journal of Ad hoc, Sensor \& Ubiquitous Computing (IJASUC) Vol.2, No.4.

[7] E-M. Royer. (2004)" A Review of Current Routing Protocols for Ad Hoc Mobile Wireless Networks",University of California, Santa Barbara Chai-Keong Toh, Georgia Institute of Technology,. Ad Hoc Networks 2, pp 1-22.

[8] G.Lavanya, A. Ebenezer Jeyakumar. (2011) "An Enhanced Secured Dynamic Source Routing Protocol for MANETS", International Journal of Soft Computing and Engineering (IJSCE), Volume $\mathrm{X}$, Issue-4.

[9] G.Lavanya, A. Ebenezer Jeyakumar. (2011) "An Enhanced Secured Dynamic Source Routing Protocol for MANETS", International Journal of Soft Computing and Engineering (IJSCE).

[10] I. Chlamtac , M.Conti, J. Liu. (2003) “Mobile ad hoc networking: imperatives and challenges", Elsevier

[11] J-E Garcia. (2003) "A novel dsr-based energy-efficient routing algorithm for mobile ad-hoc networks ", IEEE VTC-fall03.

[12] J.-E. Garcia et al. (2003) "A Novel DSR-based Energy-efficient Routing Algorithm for Mobile Adhoc Networks," in vehicular technology conference, IEEE.

[13] J. McEachen. (2012) “Wireless Ad-Hoc Networks”, Monterey, California.

[14] K. Gorantala. (2006) "Routing Protocols in Mobile Ad-hoc Networks", Master"es Thesis in Computing Science, SWEDEN.

[15] M. Aissani et al. (2007) "Optimizations and Performance Study of the Dynamic Source Routing Protocol" ThirdInternational Conference on Networking and Services(ICNS'07) IEEE.

[16] M. Rabah, N. Amina. (2006) "Routage avec différenciation de terminaux dans les réseaux mobiles ad hoc", FRANCE, RIST Vol, $16 \mathrm{n}^{\circ} 01$.

[17] M.Sreerama et al. (2011) "Performance Evalution of MANET Routing Protocols using Reference Point Group Mobility and Random WayPoint Models", International Journal of Ad hoc, Sensor \& Ubiquitous Computing (IJASUC) Vol.2, No.1.

[18] P. Padmanabhan et al,. (2008) "A survey of data replication techniques for mobile ad hoc network databases", The VLDB Journal, 17:1143-1164, Springer-Verlag.

[19] S. Chettibi and M. Benmohamed. (2009) "A Multipath Energy-Aware On demand Source Routing Protocol for Mobile Ad-Hoc Networks", 1st Workshop on Next Generation Networks: Mobility, WNGN,, Maroc.

[20] S. Kannan et al. (2011) "An optimized scheme for enhancing Mobile ad hoc Network Routing Protocol for transmitting high density data", Journal of mobile communication 5(1) :pp11-15.

[21] S. Singh et al. (1998) "Power-Aware Routing in Mobile Ad Hoc Networks," International Conference on Mobile Computing and Networking, (MobiCom'98), pp.181-190.

[22] S. Sultana et al. (2010) "Enhanced-DSR: A New Approach to Improve Performance of DSR Algorithm", Chowdhury International Journal of Computer Science and Information Technology, Volume 2, Number 2.

[23] T.Harshit et al. (2011) " A bandwidth aware weight based DSR protocol for mobile ad hoc networks", Fourth Annual ACM Bangalore Conference, USA. 
[24] T. Jiang et al. (2004) “Secure Dynamic Source Routing Protocol”, Forth International Conference on Computer and Information Technology (CIT).

[25] The Network Simulator - ns-2, http://www.isi.edu/nsnam/ns/.

[26] V.Ramesh et al. (2010) " NovelOptimization of Route Discovery in Dynamic Source Routing (DSR) Protocol for MANET, Global Journal of Computer Science and Technology”, Vol. 10 Issue 2, pp 610.

[27] W. Yu et al. (2006) “ LEARN: Localized Energy Aware Restricted Neighborhood Routing for Adhoc Networks," in Third Annual IEEE Communications Society Conference on Sensor, Mesh and Ad-hoc Communications.

\section{Authors}

\section{Mr. Sami Touil}

Actually, he is a lecturer at the department of Information Systems, College of Business and Administration at Qassim University, KSA. He received his M.Sc. in 2005 in computer science from Faculty of Sciences of Tunis, Tunisia. His B.Sc. in 2002 in management information system from the FSEG, University of Sfax, Tunisia.Mr. Touil was a lecturer in Faculty of Science of Gafsa, Tunisia from 2006 to 2008 ..

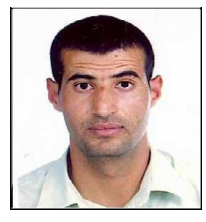

\section{Mr, Wissem Achour Chouchane}

Has got his Master in computer science in 2001 from National Engineering School of Sfax. He has joint college of business and economics at Qassim University since 2009. His M.Sc. in 2001 in computer science "Development of a supervisor with the aim of the command of a system". His research interests include in clustering in wireless ad hoc network.

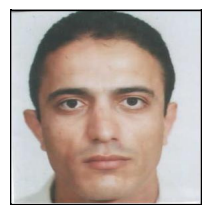

\section{Mr. Rjab Hajlaoui}

Received his Master degree in Operational Research from „FSEGS: Faculty of Economics and Management of Sfax, Tunisia in 2007. He is currently an assistant professor at the UOH, University Of Hail, Kingdom of Saudi Arabia His research interests include Wireless Sensor Network and vehicular ad hoc network.

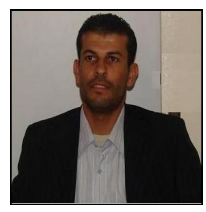

